\title{
X-raying the Star Formation History of the Universe
}

\author{
A. Cavaliere ${ }^{1}$, R. Giacconi ${ }^{2}$, and N. Menci ${ }^{3}$ \\ 1 Astrofisica, Dip. Fisica 2a Università, Roma I-00133 \\ 2 Johns Hopkins Univ., Dept. of Physics \& Astronomy, Baltimore, MD 21218 \\ ${ }^{3}$ Osservatorio Astronomico di Roma, Monteporzio, I-00044
}

\begin{abstract}
The current models of early star and galaxy formation are based upon the hierarchical growth of dark matter halos, within which the baryons condense into stars after cooling down from a hot diffuse phase. The latter is replenished by infall of outer gas into the halo potential wells; this includes a fraction previously expelled and preheated, due to momentum and energy fed back by the SNe which follow the star formation. We identify such an implied hot phase with the medium known to radiate powerful X-rays in clusters and in groups of galaxies. We show that the amount of the hot component required by the current star formation models is enough to be observable out to redshifts $z \approx 1.5$ in forthcoming deep surveys from Chandra and XMM, especially in case the star formation rate is high at such and earlier $z$. These X-ray emissions constitute a necessary counterpart, and will provide a much wanted probe of the SF process itself (in particular, of the SN feedback), to parallel and complement the currently debated data from optical and IR observations of the young stars.
\end{abstract}

Subject headings: galaxies: formation - galaxies: clusters: general - intergalactic medium - X-rays: general 


\section{Introduction}

Is there any link of the early star formation rate (SFR) with extended extragalactic sources expected to appear in the deep X-ray surveys planned (see Giacconi 1998) with Chandra and with $X M M$ ?

We expect some such connection to exist, if we carry a step further the views started by several teams (Munich: Kauffmann, White \& Guiderdoni 1993; Durham: Cole et al. 1994, Baugh et al. 1998; Santa Cruz: Somerville \& Primack 1998) to describe the processes that lead to galaxy and star formation. These are based upon the hierarchical growth of structures gravitationally dominated by dark matter (DM) halos; from the size of a galaxy to that of a galaxy group and then of a cluster, the growth occurs by repeated merging of smaller into larger structures. These views envision the baryons as condensing into stars at the minima of the forming DM potential wells, upon cooling down from a diffuse hot phase at the virial temperature of the wells.

We will show that such implied hot component - constituting, in fact, the intracluster medium (ICM) in the larger halos - yields copious bremsstrahlung emissions observable in $X$-rays. These are closely linked with the optical or infrared stellar light from which the

SFR is currently inferred. The link is provided by the energy and momentum fed back into the hot phase by the stellar winds and the supernovae (SNe) following the star formation. This process is especially relevant in the early, small but dense halos; some feedback is essential there to prevent the runaway cooling of all the contained baryons, the so-called cooling catastrophe (White \& Rees 1978).

\section{Key quantities}

SN explosions, with some contribution from stellar winds (Bressan, Chiosi \& Fagotto 1994), provide an energy output $E_{*}=E_{S N} \eta_{S N} \Delta m_{*}$; here $E_{S N} \approx 10^{51}$ erg is the energy of 
a Type II SN explosion, and $\eta_{S N} \approx 410^{-3} \Delta m_{*}$ is the combined efficiency for making SNe and massive blue stars when the mass $\Delta m_{*}$ condenses into the IMF.

Two key parameters will gauge the effectiveness of the feedback against the depth of the containing potential well. Recall that the depth, for a halo of mass $M$ with internal density $\rho \propto(1+z)^{3}$ following the background's, is marked by the circular velocity $v=\sqrt{G M / R_{v}} \propto M^{1 / 3} \rho^{1 / 6}$, or by the virial temperature $k T_{v} \propto M / R_{v} \propto v^{2}$, which takes on values around $4 \mathrm{keV}$ in rich clusters.

One parameter will gauge the dynamical effect of stars and of SN explosions by way of momentum transfer onto the surrounding gas; the resulting galactic winds can directly expel a fraction $\Delta m_{h} / m_{h} \equiv f_{*}$, especially from shallow potential wells. The proper parameter is the fractional energy converted into bulk kinetic energy at the escape velocity

$$
\epsilon_{o}=f_{*} m_{h} v^{2} / E_{*}
$$

This will take on values of order $10^{-1}$ in wells with $v \approx 150 \mathrm{~km} / \mathrm{s}$; but equally important will be its differential behavior in shallower and deeper wells.

The other parameter $T_{v} / T_{*}$ will gauge the importance of the thermal effect of the SNe. Here $k T_{v}$ measures the gravitational energy of the outer baryons, amenable to conversion into heat as they infall into the wells. The "stellar" temperature $k T_{*}=\left(1-\epsilon_{o}\right) E_{*} m_{p} / 3 \Delta m_{h}$ measures the preheating level of the expelled fraction, provided by the SNe. This may be preliminarly estimated as

$$
k T_{*}=0.7\left(1-\epsilon_{o}\right) \Delta m_{*} / \Delta m_{h} \gtrsim 0.2 \mathrm{keV},
$$

for a stellar baryonic fraction exceeding $1 / 5$, the value appropriate only for rich clusters (see Renzini 1997). But $k T_{v} \approx 0.2 \mathrm{keV}$ is also the virial value corresponding to $M \sim 510^{12} M_{\odot}$; so we may surmise that somewhere in the range from a poor group to a large galaxy stellar preheating starts to prevent the gas from piling up within the wells. Such masses begin to virialize at $z \approx 2 \pm 0.5$ in current hierarchical cosmogonies. 
We shall investigate whether more SF at early $z$, which requires more baryons condensed within the small halos, also leads to more hot gas retained and so to stronger and widespread X-ray emissions. To proceed beyond estimates we need a model for the partition, shifting with $M$ and $z$, of the baryons among the condensed, the cool, the expelled and the hot components.

\section{The model}

\subsection{Optical emissions from stars}

To that effect we start from the semi-analytic models (SAMs) developed by the teams of Munich, Durham and Santa Cruz. These include the "merging histories" of DM halos as they grow hierarchically through stochastic merging events; the above authors describe such histories using Monte Carlo simulations. We use instead the equivalent analytic probabilities provided by Bower (1991) and by Lacey \& Cole (1993), the so-called extended Press \& Schechter theory. This is because our model is considerably more complex as it

covers also the X-ray emitting baryons; so we need to cut down the computer time required to average our many observables by convolutions over the merging histories.

Along with such a rendition of the DM dynamics, the numerical package devised and built by one of us (N. Menci) includes the current SAM "recipes" to follow the condensed, the cool and the hot baryons. Here we adopt the Durham implementation where: the mass of cool gas $\Delta m_{c}$ is what can cool down at the center of the halos over their survival time; the SF from the cooling stuff obeys $\Delta m_{*}=\left[m_{c}-m_{*}-m_{h}\right] \Delta t / \tau_{*}^{o}\left(v / 300 k m s^{-1}\right)^{\alpha_{*}} ;$ the fraction returned from the cool to the hot component by galactic winds is given by $\Delta m_{h}$ $=\Delta m_{*}\left(v_{h} / v\right)^{\alpha_{h}}$, corresponding to an effective $\epsilon_{o}=\Delta m_{h} v^{2} / E_{*} \propto v^{2-\alpha_{h}}$. The luminosities of the stars so produced are computed on convolving $\dot{m}_{*}$ with the spectral energy distributions provided by the model of stellar population synthesis by Bruzual \& Charlot (1998).

The primary parameters appearing here are $\tau_{o *}, \alpha_{*}, \alpha_{h}, v_{h}$; reasonable values for these 
are known to produce reasonable first approximations to the galaxy Tully-Fisher relation, and to their colors and related luminosity functions. In closer detail, the latter are improved (as discussed in Cole et al. 1994; Somerville \& Primack 1998) on introducing secondary parameters to modulate the shape of the bright end (like the amount of absorbing dust) and the shape of the local, faint end (like the coalescence rate of the galactic baryonic cores within the halos). Such galactic parameters are implemented in our package after the Durham version, but in fact do not affect the hot diffuse ICM pervading the halos.

In this Letter we spare details and figures concerning the $\mathrm{O}$ and IR observables used here as a calibration, and refer to Menci \& Cavaliere (1999; MC99) and Poli et al. (1999). We confine ourselves to show in fig. 1 (top) our results concerning the intrinsic SFR corresponding to prompt blue stellar light. When we use the original Durham values for the parameters, we obtain optical results equal to theirs, see solid line in fig. 1 (top) and the related caption.

\subsection{X-ray emissions from the hot phase}

Our new step is the X-ray emission from the hot component (ICM) required by such SFRs. In terms of the ICM temperature $T$ and particle density $n$, the continuum luminosity reads $L_{X} \propto n^{2} R_{X}^{3} T^{1 / 2}$. Using $T \approx T_{v} \propto \rho^{1 / 3} M^{1 / 3}$ the scaling is conveniently recast as

$$
L_{X} \propto \rho^{1 / 2}\left(m_{h} / M\right)^{2}\left(n_{2} / n_{1}\right)^{2} \mathcal{I} T^{2}
$$

The shape factor $\mathcal{I}$ depends only weakly on $z$ and on halo mass $M$; it arises from the volume integration over the inner ICM profiles $n^{2}(r)$ and $T^{1 / 2}(r)$, computed (see CMT99) on assuming for the ICM hydrostatic equilibrium in the DM potential provided by Navarro, Frenk \& White (1997). The main dependences arise from expulsion and heating that affect the fraction $m_{h} / M$ of hot ICM inside the wells, and its internal density $n_{2}$ relative to the external $n_{1}$. In fact, taking constant $n_{2} / n_{1}$ and $m_{h} / M$ would give $L_{X} \propto T^{2}$, the scale-invariant relation for ICM just following the gravitational, hierarchical clustering; 
but the result would clash against the observations referred to below. To break such scale-invariance the ICM must respond actively under the drive of the stellar feedback.

The thermal feedback affects the ratio $n_{2} / n_{1}$, which depends on the strength of the accretion shocks (see CMT97) induced at about the virial radius $R_{v}$ when the outer gas falls supersonically into the halo $M$. The latter is built up hierachically by merging with, or by accretion of lumps of mass $M^{\prime}$; the associated gas is at a temperature $T^{\prime}$. Given this, the density ratio across the shock depends on its strength $T / T^{\prime}$ after the functional form $g\left(T / T^{\prime}\right)$ given by CMT99; this rises steeply from 1 when $T / T^{\prime}$ is moderately increased, but saturates to 4 for large values of $T / T^{\prime}$.

The dynamical feedback modulates this behavior, as it yields two values for $T^{\prime}$ : the "stellar" value $T_{*}^{\prime}$ applies to the fraction $f_{*}=\Delta m_{h} / m_{h}$ ejected beyond the virial radius of $M^{\prime}$ and preheated; the gravitational $T_{v}^{\prime}$ applies to the complementary $1-f_{*}$ that remains virialized inside the lump. Both such components fall into the main well, and the weighted density ratio is given by (MC99)

$$
\left(n_{2} / n_{1}\right)^{2}=f_{*} g^{2}\left(T / T_{*}^{\prime}\right)+\left(1-f_{*}\right) g^{2}\left(T / T_{v}^{\prime}\right)
$$

Meanwhile, some adiabatic compression takes place in the settling of the shocked ICM into the well. Compressions and shocks occur repeatedly during the hierarchical growth. Their balance may be expressed with a polytropic equilibrium, implying the radial profile $T(r) \propto n^{\Gamma-1}(r)$ with $1<\Gamma<1.3$ (see Cavaliere \& Fusco-Femiano 1978, Tozzi \& Norman 1999); this converts the previous history of shocks and compressions in the cluster into a space stratification. Such ICM profiles are used to compute the shape factor $\mathcal{I}$.

Averaging eq. (4) over all merging histories (i.e., over the distributions of $M^{\prime}$ ) that lead to $M$ yields the bent $L_{X}-T$ relations in fig. 1 (middle); these start steep at the scale of groups, gradually flatten, and saturate toward $L_{X} \propto T^{2}$ only in very rich clusters, in agreement with the observations by Ponman et al. (1996); Mushotzky \& Scharf (1997); Markevitch (1998); Allen \& Fabian (1998). Such a bent shape arises basically because in 
groups the stellar preheating temperature $T_{*}^{\prime}$ matches or exceeds the virial $T$ as anticipated in Sect. 2; then $T / T^{\prime}$ and $n_{2} / n_{1}$ approach 1 , so $L_{X} \propto n_{2}^{2} / n_{1}^{2}$ is weak; in fact, it is weaker when the expelled/heated fraction $f_{*}$ is larger. In rich clusters instead, $T$ exceeds any external $T_{*}^{\prime}$ and the shocks are always strong; there $n_{2} / n_{1}$ saturates to 4 , and $L_{X}$ saturates toward $L_{X} \propto T^{2}$. Additionally, a larger expelled fraction $f_{*}$ decreases the relative amount $m_{h} / M$ of ICM inside the shallow wells, and again weakens $L_{X}$ in groups.

\section{Results}

All that joins nicely with the SAMs, which are based on the same hierarchical merging histories as said above. In fact, our treatment of the hot ICM can be grafted onto the SAMs because it proceeds from the same basic equations and uses the same parameters. A double bonus of our approach is that heating the gas while expelling it outwards to low densities is a process best suited to generate in groups the high "entropy floor" observed by Ponman Cannon \& Navarro (1999); in fact, we obtain $k T_{*} / n^{2 / 3} \sim 0.510^{8 / 3} \sim 10^{2} \mathrm{keV} \mathrm{cm}^{2}$. Such a value is conserved near the center of forming clusters by the subsequent adiabatic compressions. But as a cluster forms, increasingly strong shocks develop farther out and deposit the entropy $\Delta S \propto \ln T n_{1}^{2 / 3} / T^{\prime} n_{2}^{2 / 3}$; so the entropy rises outwards, in accord with what is observed in clusters (David, Jones \& Forman 1996; Ponman et al. 1999).

The derived $L_{X}-T$ relation constitutes the intermediate step (analogous to the Tully-Fisher relation for the star light) to compute the X-ray luminosity functions and the source counts using standard formulae. We have included the line emissions using a routine updating Raymond \& Smith (1977), kindly provided by P. Tozzi.

Our fiducial cosmogony/cosmology is provided by standard cold DM initial perturbations in a flat, low-density Universe with $\Omega_{o}=0.3$ and $\Omega_{\Lambda}=0.7$, and $H_{0}=70$ $\mathrm{km} / \mathrm{s}$ Mpc. Not only this is indicated by the data concerning SNe Ia, but also it makes the baryonic fraction $\Omega_{b}=0.04$ suggested by the cosmological nucleosynthesis (see Olive 1998) 
consistent with the values $\Omega_{b} / \Omega_{0}$ around 0.15 from mass inventories in clusters (White et al. 1993; Ettori \& Fabian 1999).

So equipped, we explore the counterparts in X-rays of two extreme evolutions of the SFR for $z>1$ (shown in fig. 1, top), which bracket the currently debated shapes derived from the O-IR data. The first SFR declining for $z \gtrsim 1.5$ is obtained from the original Durham parameters (see specifically Heyl et al. 1995). Their values $\alpha_{*}=1.5$ and $\alpha_{h}=5.5$ minimize the star formation and maximize the feedback effects in the shallow, early wells; in particular, the value of $\alpha_{h}$ corresponds to $\epsilon_{o}=v^{-3}$, that is, to galactic winds stronger in shallower wells. The corresponding $L_{X}-T$ relation at $z=0,1$ is shown in the middle panel of fig. 1 (left), and the prediction for the soft X-ray counts by the solid line in the bottom panel of fig. 1 .

The other extreme evolution is the flat SFR, also shown in fig. 1 (top); this is derived from the second set of parameter values listed in the caption. The set still leads to comparable optical luminosity functions but is more in tune with recent feedback reappraisals toward the low side (see Thornton et al. 1998, Ferrara \& Tolstoy 1999; Martin 1999); it includes the "neutral" values $\alpha_{*}=0$ and $\alpha_{h}=2$. These correspond to the SF time $\tau_{*} \propto v^{-\alpha_{*}}$ and the kinetic fraction $\epsilon_{o} \propto v^{2-\alpha_{h}}$ being even for shallow and deep wells; in particular, the values of $v_{h}, \alpha_{h}$ correspond to $\epsilon_{o}=$ const $\sim 10^{-1}$ in the shallow wells, that is, to weaker winds. In the middle panel of fig. 1 (right) we show the corresponding $L_{X}-T$ relation, and by the dashed line in the bottom panel of fig. 1 the corresponding counts.

We stress that the deep counts in fig. 1 (bottom) draw increasingly apart for fluxes $F_{X}<10^{-14} \mathrm{erg} \mathrm{cm}^{-2} \mathrm{~s}^{-1}$; in fact, at fluxes 10 times fainter the counts for weak exceed those for strong winds by a factor at least 3. Following up the discussion ending §3.2, the result can be reckoned on the basis of $L_{X} \propto m_{h}^{2} n_{2}^{2} / n_{1}^{2}$ (see eqs. 3 and 4). Here $m_{h}$ $\propto f_{*}^{-1}$; in addition, $n_{2}^{2} / n_{1}^{2}$ is enhanced at low $T$ by about $f_{*}^{-1}$ for weak compared to strong feedback; so the luminosities are enhanced as $L_{X} \propto f_{*}^{-3}$ at low $T$. The numerical values of 
$f_{*}$ differ by $-20 \%$ at $L_{X} \sim 10^{43} \mathrm{erg} / \mathrm{s}$ and $z \approx 1$. In sum, with weak feedback we expect the deep counts, which rise here close to $N\left(>F_{X}\right) \propto F_{X}^{-3 / 2}$, to exceed the other case by about $(0.8)^{-9 / 2} \approx 3$ at $F_{X} \sim 10^{-15} \mathrm{erg} \mathrm{cm}^{-2} \mathrm{~S}^{-1}$, as confirmed by fig. 1 (bottom).

\section{Discussion and conclusions}

Having in mind surveys from Chandra and $X M M$ we have limited our computations to photon energies exceeeding $E_{\min }=0.25 \mathrm{keV}$, and the resolvable sources to $L_{X} \geq 10^{43} \mathrm{erg} / \mathrm{s}$ with $F_{X} \geq 10^{-15} \mathrm{erg} / \mathrm{cm}^{2} \mathrm{~s}$. So we obtain a lower bound to the divergence of the counts; this is amplified by some $20 \%$ if luminosities down to $L_{X} \sim 310^{42} \mathrm{erg} / \mathrm{s}$ are included. In the critical universe, a given $L_{X}$ would correspond to a brighter flux, to yield even larger excess counts at given flux.

Considerable uncertainties currently plague the evolution of the SFR to $z>1$, as derived from the optical (dust-obscured) and from the IR data (time-consuming to obtain, and with redshifts difficult to determine); these are discussed in detail, e.g., by Madau, Pozzetti \& Dickinson (1998), Pettini et al. (1998), Hughes et al. (1998), Barger et al. (1998), Ellis (1998). So complementary information concerning the X-ray counterparts from the forthcoming surveys planned with Chandra and XMM will be welcome and timely to address the issue. Eventually, with the O-IR data consolidating in their own right, the soft X-rays will elicit the SN feedback in action. In fact, this contributes directly to the hot component, while it only indirectly affects the stars which form from the cooling stuff; therefore the soft X-rays are best suited to probe this key quantity.

In fig. 2 we show not only the evolution of the stellar and the hot components from our computations, but also the abundances at various $z$ of the third phase constituted by the lukewarm baryons inside and outside the galaxies. These turn out to be in fair agreement with the densities (actually lower bounds) evaluated from the observations of the Ly $\alpha$ clouds seen in absorbtion, whether damped or not. 
In conclusion, a weaker feedback $\Delta m_{h} / \Delta m_{*}$ allows more baryons (hot and cold) to be retained within shallow early wells, as illustrated in fig. 2. As shown in fig. 1, such baryons yield not only a larger star formation but also stronger X-ray emissions. These involve mainly outputs $\mathrm{L}_{X} \sim 10^{43} \mathrm{erg} / \mathrm{s}$ or less at $z \approx 1$ or larger, associated with halo masses $M \sim 510^{13} M_{\odot}$ or smaller and with temperatures $T \approx 1 \mathrm{keV}$ or cooler. The deep counts in soft X-rays of souch sources and their $L_{X}-T$ relation can probe directly the feedback, the main unknown that hampers the understanding the cosmic star formation history.

We thank E. Giallongo, P. Rosati, and P. Tozzi for stimulating and informative discussions, and a referee for helpful comments. Work supported by grants from ASI and MURST.

\section{REFERENCES}

Allen, S.M, \& Fabian, A.C. 1998, MNRAS, 297, L57

Barger, A., Cowie, L.L., Sanders, D.B., \& Fulton, E. 1998, Nature, 394, 248

Baugh, C.M., Cole, S., Frenk, C.S., \& C.G. Lacey 1998, ApJ, 498, 504

Bower, R. 1991, MNRAS, 248, 332

Bressan, A, Chiosi, C, \& Fagotto, F. 1994, ApJ SS, 94, 63

Bruzual, A., \& Charlot, S. 1998, update of ——1993, ApJ, 405, 538

Cavaliere, A, \& Fusco Femiano, R. 1978, A\&A, 70, 767

Cavaliere, A., Menci, N., Tozzi, P., 1997, ApJL 484, 1 (CMT97)

-1999, preprint astro-ph9810498

Cole, S., Aragon-Salamanca, A., Frenk, C.S., Navarro, J.F., \& Zepf, S.E. 1994, MNRAS, 271,781

David, L.P., Jones, C., \& Forman, W. 1996, ApJ, 473, 692 
Ettori, S., \& Fabian, A.C. 1999, MNRAS, 305, 843

Ellis, R. 1998, Nature, 395, 3

Ferrara, A., \& Tolstoy, R. 1999, preprint astro-ph/9905280

Giallongo, E., Fontana, A., \& Madau, P. 1997, MNRAS, 289, 629

Giacconi, R. 1998, Astron. Nachr. 319, 147

Kauffmann, G., White, S.D.M., \& Guiderdoni, B. 1993, MNRAS, 264, 201

Heyl, J.S., Cole, S., Frenk, C.S., \& Navarro, J.F. 1995, MNRAS, 274, 755

Hughes, D. et al. 1998, Nature, 394, 241 ]

Lacey, C., \& Cole, S. 1993, MNRAS, 262, 627

Madau, P., Pozzetti, L., \& Dickinson, M.E. 1998, ApJ, 498, 106

Markevitch, M. 1998, ApJ, 504, 27

Martin, C. 1999, ApJ, 513, 156

Menci, N., \& Cavaliere, A. 1999, MNRAS, in press astro-ph/9909259

Mushotzky, R.F., \& Scharf, C.A., 1997, ApJ, 482, 13

Navarro, J.F., Frenk, C.S., \& White, S.D.M. 1997, ApJ, 490, 493

Olive, K.A. 1998, in Proc. of 19th Texas Symposium on Relativistic Astrophysics and Cosmology, eds. J. Paul et al. (Saclay: CEA)

Pettini, M., Kellog, M., Steidel, C.C., Dickinson, M., Adelberger, K.L., Giavalisco, M. 1998, ApJ, 508, 539

Ponman, T.J., Bourner, P.D.J., Ebeling, H., Böhringer, H. 1996, MNRAS, 283, 690

Ponman, T.J., Cannon, D.B., \& Navarro, J.F. 1999, Nature, 397, 135

Poli, F., Giallongo, E., Menci, N., D’Odorico, S., \& Fontana, A. 1999, ApJ, in press astro-ph/9910537 
Raymond, J.C., \& Smith, B.W. 1977, ApJS, 35, 419

Renzini, A. 1997, ApJ 488, 35

Rosati, P., Della Ceca, R., Norman, C., \& Giacconi, R. 1998, ApJ, 492, 21

Somerville, R.S., \& Primack, J.R. 1998, preprint astro-ph/9802269

Tozzi, P. \& Norman, C. 1999, preprint astro-ph9905046

Thornton, K., Gaudlitz, M., Janka, H.-Th., Steinmetz, M. 1998, ApJ, 500, 95

White, S.D.M., Navarro, J.F., Frenk, C.S., \& Evrard, A.E. 1993, Nature, 366, 429

White, S.D.M., \& Rees, M.J. 1978, MNRAS, 183, 341 


\section{FIGURE CAPTIONS}

Fig. 1.- Top: The $z$-dependent SFR from our model of baryon processing during the hierarchical clustering in the flat universe with $\Omega_{o}=0.3, H_{o}=70 \mathrm{~km} / \mathrm{s} \mathrm{Mpc}$. The solid line represents the shape peaked at $z \approx 1.5$ resulting from the Durham set of parameters: $\tau_{*}^{o}=2$ Gyr, $\alpha_{*}=-1.5, v_{h}=140 \mathrm{~km} / \mathrm{s}, \alpha_{h}=5.5$, implying strong galactic winds. The dashed line represents the SFR flat toward high $z$ resulting from our "neutral" set: $\tau_{*}^{0}=2 \mathrm{Gyr}$, $\alpha_{*}=0, v_{h}=140 \mathrm{~km} / \mathrm{s}, \alpha_{h}=1.5$, implying weak winds. Middle: The $L_{X}-T$ correlations at $z=0$ (solid line) and at $z=1$ (dotted line) corresponding to the peaked (left frame) and to the flat SFR (right frame) illustrated on top. Group data from Ponman et al. (1996, solid squares); cluster data from Markevitch (1998, open circles). Bottom: The source counts $N\left(>F_{X}\right)$ corresponding to the peaked SFR (solid line) and to the flat SFR (dashed line), in the energy range $0.25-2 \mathrm{keV}$. The hatched band represents the cluster number counts observed by Rosati et al. (1998).

Fig. 2.- The contribution to the baryon density $\Omega_{b}$ from the lukewarm baryons with $T \leq 510^{4} \mathrm{~K}$, from the hot virialized ICM and from the stars, see labels in the figure. The solid lines show the model predictions corresponding to strong winds, while the dashed lines correspond to weak winds. The density of lukewarm baryons is compared with the data (from Giallongo et al. 1997) concerning the density of photoionized intergalactic gas, which constitute lower limits especially for $z<3$. 

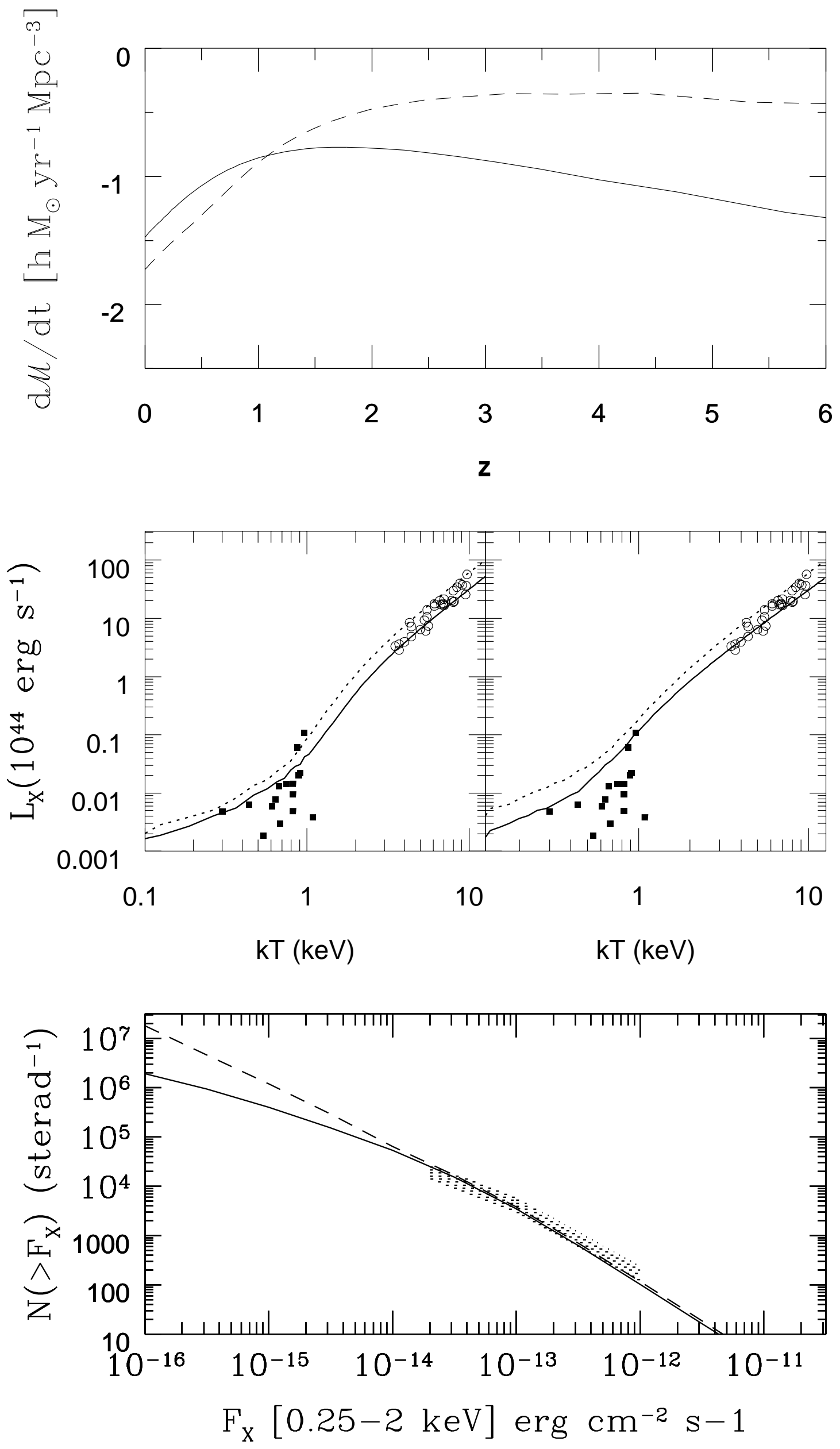


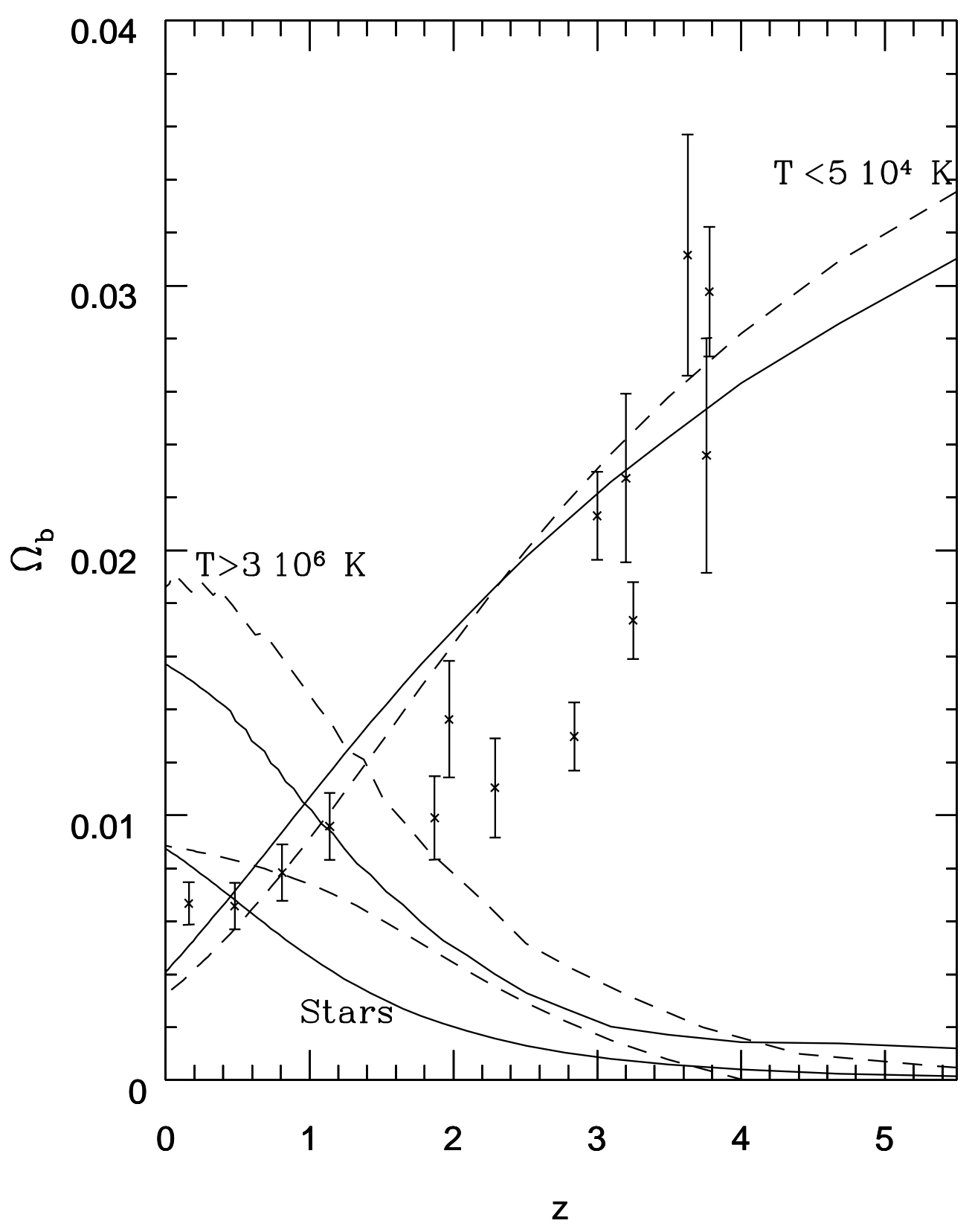

